\title{
Closure Systems as a Fuzzy Extension of Meet-subsemilattices
}

\author{
Manuel Ojeda-Hernández, Inma P. Cabrera, Pablo Cordero and Emilio Muñoz-Velasco \\ Dept. of Applied Mathematics, University of Malaga, Spain \\ \{manuojeda, ipcabrera, pcordero, ejmunoz\}@uma.es
}

\begin{abstract}
An extension of the notion of closure system is studied adapting the idea of meetsubsemilattice to a complete fuzzy lattice. Results relating closure operators and closure systems in the classical case are extended properly to this framework. This definition is proved to be equivalent to the most used definition given by Bělohlávek on the fuzzy powerset lattice. A definition of fuzzy closure system is presented and related to closure systems.
\end{abstract}

\section{Introduction}

Closure operators have an important role in pure, applied mathematics and computer science [10]. Fuzzy closure operators [1, 4] appear in several areas of fuzzy logic and its applications, including fuzzy mathematical morphology [13, 18], fuzzy relational equations [12], approximate reasoning [6, 11] and fuzzy logic in narrow sense [15], and its applications such as fuzzy logic programming [17] or formal concept analysis of data with fuzzy attributes [20].

As in the classical case, there is a bijection which relates the notions of closure operator and closure system. We can find distinct definitions of closure system depending on the ordered structure on which the fuzzy closure operator is defined. As a consequence, the notion of fuzzy closure system has been defined on $L$-ordered sets [14], on fuzzy preposets [8] and fuzzy preordered structures [9].

This paper is a continuation on the study of fuzzy closure systems done in [19], where the underlying structure was a Heyting algebra. Our aim is to study the concept of closure system on complete fuzzy lattices. One can find several extensions of the notion of lattice and complete lattice to the fuzzy framework. Basically, these extensions go in two main ways.

On the one hand, some authors define a fuzzy lattice as a fuzzy subset of a (classical) lattice $L$ that is compatible with the structure of the lattice in some manner [21, 23]. This particular extension could be called that of fuzzy sublattices. Closure systems on fuzzy sublattices were introduced in [22].

On the other hand, sometimes a fuzzy lattice is defined as a (crisp) set endowed with a fuzzy order relation satisfying certain conditions related to the existence of generalized infimum and supremum. The concept of complete fuzzy lattice considered in this work belongs to this class. It was introduced in [5] with the name of completely lattice L-ordered set, although it has been called other names, for instance, fuzzy complete lattice in [16]. A particular case of fuzzy complete lattice is the $L$-powerset with the so-called subsethood degree relation $S$. Closure systems on $L$-powerset lattices were introduced by Bělohlávek in [1].

The starting point of this paper is the definition of closure system, in the framework of complete fuzzy lattices, provided in [3]. This extension is inspired by the notion of closure system in the case of classical complete lattices, where they are $\wedge$-subsemilattices. As awaited, the relationship between fuzzy closure operators and closure systems is studied. After a first section of preliminaries, the second section includes the definition of closure system in a fuzzy framework, which extends the classical closure system property of being a $\wedge$-subsemilattice. A one-to-one relation between closure systems and fuzzy closure operators is proved, i.e., closure systems induce closure operators and closure operators induce closure systems in a desirable manner. In addition, a characterization of closure system in terms of the minimum of certain sets is proved. The core of the next section is the study of the relationship between the definition of closure system and the one of $L$-closure system given by Bělohlávek 
in [1]. The main result within this section is the equivalence of both definitions on $L$-powerset lattices. Furthermore, the notion of fuzzy closure system in this framework, which is not as straightforward as the one on Heyting algebras provided in [19], is introduced and its good properties are shown. In the last section, the conclusions of our research are shown and some hints of future work are presented.

\section{Preliminaries}

The framework to which we are going to generalize the notion of closure system is introduced in this section. It has been chosen with the idea of being as general as possible and thus have a wider range of possible applications. Specifically, we recall the notions of complete residuated lattices [2, 15], fuzzy poset and fuzzy complete lattice, and some basics results that will be needed throughout the paper [5, 16].

Throughout this paper, let $\mathbb{L}=(L, \wedge, \vee, \otimes, \rightarrow, 0,1)$ be a complete residuated lattice, which is an algebra where

- $(L, \wedge, \vee, 0,1)$ is a complete lattice with 0 and 1 being the least and the greatest elements of $L$, respectively,

- $(L, \otimes, 1)$ is a commutative monoid (i.e., $\otimes$ is commutative, associative, and 1 is neutral with respect to $\otimes)$, and

- $\otimes$ and $\rightarrow$ satisfy the so-called adjointness property: for all $a, b, c \in L$, we have that $a \otimes b \leq c$ iff $a \leq b \rightarrow c$.

This structure is utilized in mathematical fuzzy logics and their applications as structures of truth degrees with $\otimes$ and $\rightarrow$ used as truth functions of fuzzy conjunction and fuzzy implication, respectively [15]. The unit interval with the Łukasiewicz, Gödel and Goguen pairs of t-norms and implications are examples of residuated complete lattices.

An $\mathbb{L}$-set is a mapping $X: U \rightarrow L$ from the universe set $U$ to the membership values set $L$, where $X(u)$ means the degree in which $u$ belongs to $X$. The set of $\mathbb{L}$-sets on the universe $U$ is denoted by $L^{U}$. A crisp set is considered to be a particular case of $\mathbb{L}$-set by using its characteristic mapping $X: U \rightarrow\{0,1\}$ with $X(u)=1$ iff $u \in X$.

Operations with $\mathbb{L}$-sets are defined element-wise. For instance, $A \otimes B \in L^{U}$ is defined as $(A \otimes B)(u)=A(u) \otimes$ $B(u)$ for all $u \in U$. The so-called subsethood degree relation is defined as $S: L^{U} \times L^{U} \rightarrow L$ where

$$
S(A, B)=\bigwedge_{x \in U}(A(x) \rightarrow B(x)) .
$$

Obviously, $S(A, B)=1$ if and only if $A(x) \leq B(x)$ for all $x \in U$. In this case, $A$ is said to be a fuzzy subset of $B$ and is denoted by $A \subseteq B$.

Binary $\mathbb{L}$-relations (binary fuzzy relations) on a set $U$ can be thought of as $\mathbb{L}$-sets on the universe $U \times U$. That is, a binary $\mathbb{L}$-relation on $U$ is a mapping $\rho \in L^{U \times U}$ assigning to each $x, y \in U$ a truth degree $\rho(x, y) \in L$ (a degree to which $x$ and $y$ are related by $\rho)$.

For $\rho$ being a binary $\mathbb{L}$-relation in $U$, we say that

- $\rho$ is reflexive if $\rho(x, x)=1$ for all $x \in U$.

- $\rho$ is symmetric if $\rho(x, y)=\rho(y, x)$ for all $x, y \in U$.

- $\rho$ is antisymmetric if $\rho(x, y) \otimes \rho(y, x)=1$ implies $x=y$ for all $x, y \in U$.

- $\rho$ is transitive if $\rho(x, y) \otimes \rho(y, z) \leq \rho(x, z)$ for all $x, y, z \in U$.

Definition 1. Given a non-empty set $A$ and a binary $\mathbb{L}$-relation $\rho$ on $A$, the pair $\mathbb{A}=(A, \rho)$ is said to be a

- fuzzy preposet if $\rho$ is a fuzzy preorder, i.e. if $\rho$ is reflexive and transitive;

- fuzzy poset if $\rho$ is a fuzzy order, i.e. if $\rho$ is reflexive, antisymmetric and transitive.

A typical example of fuzzy poset is $\left(L^{U}, S\right)$.

As in the crisp case, any order $\mathbb{L}$-relation implicitly defines an equivalence $\mathbb{L}$-relation that is called symmetric kernel relation. In the fuzzy case, this equivalence $\mathbb{L}$ relation usually replaces the notion of equality in the fuzzy poset.

Definition 2. Given a fuzzy preposet $\mathbb{A}=(A, \rho)$, the symmetric kernel relation is defined as $\approx: A \times A \rightarrow L$ where $(a \approx b)=\rho(a, b) \wedge \rho(b, a)$ for all $a, b \in A$.

Proposition 3. Given a fuzzy preposet $\mathbb{A}=(A, \rho)$, the symmetric kernel relation $\approx$ is a fuzzy equivalence relation, that is, it is a reflexive, symmetric and transitive fuzzy relation.

A usual way to define fuzzy algebras is to consider as an underlying structure a pair which consists of a set and a tolerance or equivalence relation on it. Thus, an alternative definition of fuzzy poset that can be found in the literature [9] is given by a tuple $(A, \approx, \rho)$ where $\approx$ is a fuzzy equivalence relation on $A$ and $\rho$ is a fuzzy order that is compatible with $\approx$. In [24], it is shown that both definitions of fuzzy poset are equivalent. 
To present the notion of fuzzy lattice we need to generalize those of upper (lower) bound and supremum (infimum).

Definition 4. Given a fuzzy preposet $\mathbb{A}=(A, \rho)$ and a fuzzy set $X \in L^{A}$, we define the down-cone of $X$ and the up-cone of $X$, respectively, as the fuzzy sets $X_{\rho}, X^{\rho} \in$ $L^{A}$ where, for all $a \in A$,

$$
\begin{aligned}
& X_{\rho}(a)=\bigwedge_{x \in A}(X(x) \rightarrow \rho(a, x)) \quad \text { and } \\
& X^{\rho}(a)=\bigwedge_{x \in A}(X(x) \rightarrow \rho(x, a)) .
\end{aligned}
$$

Thus, $X_{\rho}(a)$ and $X^{\rho}(a)$ can be seen as the degree to which $a$ is an upper bound and lower bound of $X$, respectively.

Notice that for singletons, $X=\{x\}$, we will omit the brackets for simplicity of the notation, thus $x^{\rho}(a)=$ $\rho(x, a)$ and $x_{\rho}(a)=\rho(a, x)$.

Definition 5. Let $\mathbb{A}=(A, \rho)$ be a fuzzy preposet and a fuzzy subset $X$ of $A$.

An element $m \in A$ is a $p$-minimum of $X$ iff

i. $X(m)=1$, and

ii. $X \subseteq m^{\rho}$, i.e., $X(x) \leq \rho(m, x)$, for all $x \in A$.

An element $M \in A$ is a $p$-maximum of $X$ iff

i. $X(M)=1$, and

ii. $X \subseteq M_{\rho}$, i.e., $X(x) \leq \rho(x, M)$, for all $x \in A$.

The crisp set of p-minima (resp. p-maxima) for $X$ will be denoted $\mathrm{p}-\min (X)$ (resp. $\mathrm{p}-\max (X))$. Notice that, due to the absence of antisymmetry, this set could have more than one element. If $\mathbb{A}$ is a poset, then every non-empty set of p-minima and p-maxima is a singleton whose element will be called the minimum (resp. maximum) of $X$, denoted by $\min (X)($ resp. $\max (X))$.

Definition 6. Let $\mathbb{A}=(A, \rho)$ be a fuzzy preposet and $X \in L^{A}$. An element $a \in A$ is said to be infimum (resp. supremum) of $X$ if the following conditions hold:

i. $X_{\rho}(a)=1\left(\operatorname{resp} . X^{\rho}(a)=1\right)$.

ii. $X_{\rho}(x) \leq \rho(x, a)$ (resp. $\left.X^{\rho}(x) \leq \rho(a, x)\right)$, for all $x \in A$.

The following propositions, which are straightforward, provide useful characterizations of infimum, supremum, p-minimum and p-maximum.
Proposition 7. Let $\mathbb{A}=(A, \rho)$ be a fuzzy preposet and $X \in L^{A}$. An element $a \in A$ is infimum (resp. supremum) of $X$ if and only if, for all $x \in A$,

$$
\rho(x, a)=X_{\rho}(x) \quad\left(\text { resp. } \rho(a, x)=X^{\rho}(x)\right) .
$$

Proposition 8. Let $\mathbb{A}=(A, \rho)$ be a fuzzy preposet and $X \in L^{A}$. An element $m \in A$ is a p-minimum (resp. pmaximum) of $X$ if and only if $m$ is an infimum (resp. a supremum) of $X$ and $X(m)=1$.

Notice that the supremum (resp. infimum) needs not be unique in an arbitrary preposet, but if $\mathbb{A}$ is a poset and due to antisymmetry, it is not difficult to see that, if a supremum (resp. infimum) of $X$ exists, it is unique. In such case, we will denote it by $\bigsqcup X$ (resp. $\sqcap X$ ).

Definition $9([5])$. We say that a fuzzy poset $(A, \rho)$ is a complete fuzzy lattice if every fuzzy subset $X \in L^{A}$ has supremum and infimum.

The pair $\left(L^{U}, S\right)$ is an example of complete fuzzy lattice, which is called the L-powerset lattice of $U$. This fact follows easily from [2, Theorem 5.63].

Notice that, if $(A, \rho)$ is a complete fuzzy lattice, then $(A, \leq)$, with $a \leq b$ iff $\rho(a, b)=1$, is a complete lattice. Therefore, there exist elements that are minimum and maximum, which we denote by $\perp$ and $T$ respectively.

We conclude this section with the usual definition of closure operator on a fuzzy preposet.

Definition 10. Given a fuzzy preposet $\mathbb{A}=(A, \rho)$, a mapping c: $A \rightarrow A$ is said to be a closure operator on $\mathbb{A}$ if the following conditions hold:

i. $\rho(a, b) \leq \rho(\mathrm{c}(a), \mathrm{c}(b))$, for all $a, b \in A$

ii. $\rho(a, \mathrm{c}(a))=1$, for all $a \in A$

iii. $\rho(\mathrm{c}(\mathrm{c}(a)), \mathrm{c}(a))=1$, for all $a \in A$.

Conditions i and ii are well-known and are called isotony and inflationarity, respectively. Observe that condition iii could be replaced by $(\mathrm{c}(\mathrm{c}(a)) \approx$ $\mathrm{c}(a))=1$, and, thus, if $\mathbb{A}$ is a fuzzy poset, a closure operator is idempotent in a classical sense, i.e., $\mathrm{c}(\mathrm{c}(a))=\mathrm{c}(a)$ for all $a \in A$.

\section{Closure systems on fuzzy lattices}

A generalization of the classical definition of closure system on complete lattices was proposed in [3], where they are considered as sets with the $\wedge$-subsemilattice structure.

Definition 11. Let $(A, \rho)$ be a fuzzy complete lattice. A crisp subset $\mathscr{F} \subseteq A$ is said to be a closure system if $\prod X \in \mathscr{F}$ for any fuzzy subset $X$ of $\mathscr{F}$. 
Notice that the empty set is contained in every set and $\Pi \varnothing=\top$. Therefore, $\top$ is in every closure system.

Example 1. Let $\mathbb{L}=(\{0,0.5,1\}, \wedge, \vee, \otimes, \rightarrow, 0,1)$ be the three-valued Łukasiewicz residuated lattice, and $(A, \rho)$ be the fuzzy lattice where $A=\{\perp, a, b, c, d, e, \top\}$ and $\rho: A \times A \rightarrow L$ is the fuzzy relation order described by the following table:

\begin{tabular}{c|ccccccc}
$\rho$ & $\perp$ & $a$ & $b$ & $c$ & $d$ & $e$ & $\top$ \\
\hline$\perp$ & 1 & 1 & 1 & 1 & 1 & 1 & 1 \\
$a$ & 0.5 & 1 & 0.5 & 1 & 1 & 1 & 1 \\
$b$ & 0.5 & 0.5 & 1 & 1 & 1 & 1 & 1 \\
$c$ & 0.5 & 0.5 & 0.5 & 1 & 1 & 1 & 1 \\
$d$ & 0 & 0.5 & 0 & 0.5 & 1 & 0.5 & 1 \\
$e$ & 0 & 0 & 0.5 & 0.5 & 0.5 & 1 & 1 \\
$\top$ & 0 & 0 & 0 & 0.5 & 0.5 & 0.5 & 1 \\
\hline
\end{tabular}

The set $\mathscr{F}=\{c, \top\}$ is a closure system because, for any fuzzy subset $X$ of $\mathscr{F}$, we have that $\prod X=c$ when $X(c)=1$, and $\prod X=\top$ otherwise.

Now we provide a proof for the following theorem that was stated in [3]. It shows the existence of a bijection between closure systems and closure operators, as in the classical case.

Theorem 12. Let $\mathbb{A}=(A, \rho)$ be a complete fuzzy lattice.

i. If $\mathscr{F}$ is a closure system on $\mathbb{A}$, then the mapping $\mathrm{c}_{\mathscr{F}}: A \rightarrow A$ defined as $\mathrm{c}_{\mathscr{F}}(x)=\prod\left(x^{\rho} \cap \mathscr{F}\right)$ is a closure operator on $\mathbb{A}$.

ii. If $\mathrm{c}: A \rightarrow A$ is a closure operator on $\mathbb{A}$, then $\mathscr{F}_{\mathrm{c}}=$ $\{x \in A \mid \mathrm{c}(x)=x\}$ is a closure system on $\mathbb{A}$.

iii. If $\mathscr{F}$ is a closure system on $\mathbb{A}$, then $\mathscr{F}_{\mathrm{c}_{\mathscr{F}}}=\mathscr{F}$.

iv. If $\mathrm{c}: A \rightarrow A$ is a closure operator on $\mathbb{A}$, then $\mathrm{c} \mathscr{F}_{\mathrm{c}}=\mathrm{c}$.

Proof. First, assume that $\mathscr{F}$ is a closure system and, therefore, $\mathrm{c}_{\mathscr{F}}(x) \in \mathscr{F}$ for all $x \in A$.

$$
\begin{array}{r}
\rho(x, \mathrm{c} \mathscr{F}(x))=\rho\left(x, \prod\left(x^{\rho} \cap \mathscr{F}\right)\right)= \\
=\left(x^{\rho} \cap \mathscr{F}\right)_{\rho}(x)=\bigwedge_{a \in A}\left[\left(x^{\rho} \cap \mathscr{F}\right)(a) \rightarrow \rho(x, a)\right]
\end{array}
$$

Since $\left(x^{\rho} \cap \mathscr{F}\right)(a)$ is $\rho(x, a)$ if $a \in \mathscr{F}$ and 0 otherwise, we get,

$$
\begin{array}{r}
\bigwedge_{a \in A}\left[\left(x^{\rho} \cap \mathscr{F}\right)(a) \rightarrow \rho(x, a)\right]= \\
=\bigwedge_{a \in \mathscr{F}}[\rho(x, a) \rightarrow \rho(x, a)]=1, \text { for all } x \in A
\end{array}
$$

Hence, $\rho\left(x, \mathrm{c}_{\mathscr{F}}(x)\right)=1$, for all $x \in A$, i.e. $\mathrm{c}_{\mathscr{F}}$ is inflationary.
By the properties of infima, for all $a \in A$ and $X \in L^{A}$, we have $X(a) \leq \rho\left(\prod X, a\right)$. In particular, for $X=x^{\rho} \cap$ $\mathscr{F}$, we have that

$$
\begin{array}{r}
\rho(x, v)=\left(x^{\rho} \cap \mathscr{F}\right)(v) \leq \rho\left(\mathrm{c}_{\mathscr{F}}(x), v\right), \\
\text { for all } x \in A, v \in \mathscr{F} .
\end{array}
$$

Now, for any $x, y \in A$, since $\mathrm{c}_{\mathscr{F}}$ is inflationary and $\mathrm{c}_{\mathscr{F}}(y) \in \mathscr{F}$, it follows,

$$
\begin{array}{r}
\rho(x, y)=\rho(x, y) \otimes \rho\left(y, \mathrm{c}_{\mathscr{F}}(y)\right) \leq \\
\leq \rho\left(x, \mathrm{c}_{\mathscr{F}}(y)\right) \leq \rho\left(\mathrm{c}_{\mathscr{F}}(x), \mathrm{c}_{\mathscr{F}}(y)\right) .
\end{array}
$$

Therefore, $\mathrm{c}_{\mathscr{F}}$ is isotone.

Let $x \in A$. Since, by hypothesis, $\mathrm{c}_{\mathscr{F}}(x) \in \mathscr{F}$, and by using (1), we get

$$
1=\rho\left(c_{\mathscr{F}}(x), c_{\mathscr{F}}(x)\right) \leq \rho\left(c_{\mathscr{F}}\left(c_{\mathscr{F}}(x)\right), c_{\mathscr{F}}(x)\right) .
$$

Therefore, $\rho\left(c_{\mathscr{F}}\left(\mathrm{c}_{\mathscr{F}}(x)\right), \mathrm{c}_{\mathscr{F}}(x)\right)=1$, for all $x \in A$, i.e. $c_{\mathscr{F}}$ is idempotent; and we have proced that it is a closure operator.

For ii, assume $\mathrm{c}$ is a closure operator in $(A, \rho)$ and let $\mathscr{F}_{\mathrm{c}}=\{x \in A \mid \mathrm{c}(x)=x\}$. For every fuzzy subset $X \subseteq$ $\mathscr{F}_{c}$, we denote $m=\prod X$ and prove $m \in \mathscr{F}_{c}$.

By Proposition 7, we have that

$$
\begin{aligned}
& \rho(\mathrm{c}(m), m)=X_{\rho}(\mathrm{c}(m))= \\
& =\bigwedge_{a \in A}(X(a) \rightarrow \rho(\mathrm{c}(m), a))
\end{aligned}
$$

Now, since $X \subseteq \mathscr{F}_{\mathrm{c}}$, we have that $X(a)=0$ for all $a \notin$ $\mathscr{F}_{\mathrm{c}}$ and, by using it and the fact that $\mathrm{c}(s)=s$ for all $s \in \mathscr{F}_{\mathrm{c}}$, we obtain

$$
\begin{aligned}
\rho(\mathrm{c}(m), m) & =\bigwedge_{s \in \mathscr{F}_{\mathrm{c}}}(X(s) \rightarrow \rho(\mathrm{c}(m), s))= \\
& =\bigwedge_{s \in \mathscr{F}_{\mathrm{c}}}(X(s) \rightarrow \rho(\mathrm{c}(m), \mathrm{c}(s)))
\end{aligned}
$$

Therefore, $\rho(\mathrm{c}(m), m)=1$ because, since $m$ is the infimum of $X$ and $c$ is isotone, we have $X(s) \leq \rho(m, s) \leq$ $\rho(\mathrm{c}(m), \mathrm{c}(s))$. Finally, since $\mathrm{c}$ is inflationary, and by antysimmetry, we get $m=\mathrm{c}(m) \in \mathscr{F}_{c}$.

Item iii is proved by double inclusion. On the one hand, if $s \in \mathscr{F}$, then by the first property of the infimum

$$
1=\rho(s, s)=\left(s^{\rho} \cap \mathscr{F}\right)(s) \leq \rho\left(\mathrm{c}_{\mathscr{F}}(s), s\right) .
$$

Also, by inflationarity of the closure operator we had $\rho\left(s, c_{\mathscr{F}}(s)\right)=1$. Hence, using antisymmetry $\rho\left(s, \mathrm{c}_{\mathscr{F}}(s)\right) \otimes \rho\left(\mathrm{c}_{\mathscr{F}}(s), s\right)=1$ implies $s=\mathrm{c}_{\mathscr{F}}(s)$. Then, $s \in \mathscr{F}_{c_{\mathscr{F}}}$ and we have proved $\mathscr{F} \subseteq \mathscr{F}_{\mathrm{c}_{\mathscr{F}}}$.

On the other hand, if $s \in \mathscr{F}_{\mathrm{c}_{\mathscr{F}}}$, this means $\rho\left(\mathrm{c}_{\mathscr{F}}(s), s\right)=1$. By inflationarity we have 
$\rho\left(s, \mathrm{c}_{\mathscr{F}}(s)\right)=1$. Hence by antisymmetry $s=\mathrm{c}_{\mathscr{F}}(s)$ and by definition of $\mathrm{c}_{\mathscr{F}}, s=\mathrm{c}_{\mathscr{F}}(s) \in \mathscr{F}$. Thus, $\mathscr{F}_{\mathrm{c} \mathscr{F}} \subseteq \mathscr{F}$.

In order to prove item iv, i.e. $\mathrm{c}(x)=\mathrm{c}_{\mathscr{F}_{\mathrm{c}}}(x)$, it suffices to show $\mathrm{c}(x)$ is the infimum of $\left(x^{\rho} \cap \mathscr{F}_{\mathrm{c}}\right)$.

First, for all $y \in A$, we have

$$
\begin{gathered}
\left(x^{\rho} \cap \mathscr{F}_{\mathrm{c}}\right)_{\rho}(y)=\bigwedge_{a \in A}\left[\left(x^{\rho} \cap \mathscr{F}_{\mathrm{c}}\right)(a) \rightarrow \rho(y, a)\right]= \\
=\bigwedge_{a \in \mathscr{F}_{\mathrm{c}}}[\rho(x, a) \rightarrow \rho(y, a)] \\
\leq \rho(x, \mathrm{c}(x)) \rightarrow \rho(y, \mathrm{c}(x))=\rho(y, \mathrm{c}(x)) .
\end{gathered}
$$

Moreover, given $y \in A$ and $a \in \mathscr{F}_{\mathrm{c}}$, we obtain

$$
\rho(y, \mathrm{c}(x)) \leq \rho(\mathrm{c}(x), a) \rightarrow \rho(y, a) \stackrel{1}{\leq} \rho(x, a) \rightarrow \rho(y, a)
$$

and, since it is true for all $a \in \mathscr{F}_{\mathrm{c}}$, we have that

$$
\rho(y, \mathrm{c}(x)) \leq \bigwedge_{a \in \mathscr{F}_{\mathrm{c}}}[\rho(x, a) \rightarrow \rho(y, a)]=\left(x^{\rho} \cap \mathscr{F}_{\mathrm{c}}\right)_{\rho}(y) .
$$

Thus, by Proposition 7, $\mathrm{c}(x)=\prod\left(x^{\rho} \cap \mathscr{F}_{\mathrm{c}}\right)$, for all $x \in$ $A$ and $\mathrm{c}=\mathrm{c}_{\mathscr{F}_{\mathrm{c}}}$ is proved.

The previous theorem establishes a bijection between closure systems and closure operators in complete fuzzy lattices. Given a closure operator c : $A \rightarrow A$, the set $\mathscr{F}_{\mathrm{c}}=\{x \in A \mid \mathrm{c}(x)=x\}$ is the closure system associated to $\mathrm{c}$, and, given a closure system $\mathscr{F}$, the mapping $\mathrm{c}_{\mathscr{F}}: A \rightarrow A$ defined as $\mathrm{c}_{\mathscr{F}}(x)=\prod\left(x^{\rho} \cap \mathscr{F}\right)$ is the closure operator associated to $\mathscr{F}$. This last construction leads to a characterization of closure systems in terms of minima.

Proposition 13. Let $\mathbb{A}=(A, \rho)$ be a complete fuzzy lattice. A crisp set $\mathscr{F} \subseteq A$ is a closure system if and only if the element $\min \left(x^{\rho} \cap \mathscr{F}\right)$ exists for all $x \in A$.

Proof. Assume $\mathscr{F}$ is a closure system and let $x \in A$. Consider the set $x^{\rho} \cap \mathscr{F}$, which is a subset of $\mathscr{F}$. Since $\mathscr{F}$ is a closure system, $\prod\left(x^{\rho} \cap \mathscr{F}\right)$ is an element of $\mathscr{F}$. Furthermore, from Theorem 12, we have that $\rho\left(x, \prod\left(x^{\rho} \cap \mathscr{F}\right)\right)=1$ and $\left(x^{\rho} \cap \mathscr{F}\right)\left(\prod\left(x^{\rho} \cap \mathscr{F}\right)\right)=1$, so it is the minimum of the set.

Conversely, assume the set $\mathscr{F}$ is such that for all $x \in A$, $\min \left(x^{\rho} \cap \mathscr{F}\right)$ exists.

Let $X \subseteq \mathscr{F}$ and consider the set $(\Pi X)^{\rho} \cap \mathscr{F}$. Following the assumption, the element $m=\min \left((\Pi X)^{\rho} \cap \mathscr{F}\right)$ exists. We claim $m=\prod X$.

By definition of minimum, $\left(\left(\prod X\right)^{\rho} \cap \mathscr{F}\right)(m)=1$, i.e., $\rho\left(\prod X, m\right)=1$ and $m \in \mathscr{F}$.
By definition of infimum, $X(x) \leq \rho(\Pi X, x)$, for all $x \in$ $A$ and $X_{\rho}(x) \leq \rho\left(x, \prod X\right)$, for all $x \in A$.

$$
X_{\rho}(m)=\bigwedge_{x \in A} X(x) \rightarrow \rho(m, x) \stackrel{X \subseteq \mathscr{F}}{=} \bigwedge_{s \in \mathscr{F}} X(s) \rightarrow \rho(m, s) .
$$

Let $s \in \mathscr{F}$, then

$$
X(s) \leq \rho\left(\prod X, s\right)=\left(\prod X^{\rho} \cap \mathscr{F}\right)(s) \leq \rho(m, s)
$$

Hence, $X_{\rho}(m)=1 \leq \rho\left(m, \prod X\right)$, so $\prod X=m \in \mathscr{F}$.

\section{Equivalence with L-closure systems in powerset lattices}

The most cited definition of closure system in a fuzzy setting was given by Bělohlávek in [1], where the notions of $L_{K}$-closure operator and $L_{K}$-closure system on $\mathbb{L}$-ordered sets were introduced, $K$ being a filter of the residuated lattice $\mathbb{L}$. Moreover, the product and residuum of $\mathbb{L}$ have been extended to an external operation on the powerset lattice as follows:

$$
\begin{array}{r}
(a \otimes A)(x)=a \otimes A(x) \text { and } \\
(a \rightarrow A)(x)=a \rightarrow A(x) .
\end{array}
$$

for all $a, x \in L, A \in L^{U}$.

Notice that these operations involve elements of $L$ and elements of $L^{U}$, hence they are particularly defined on the powerset lattice.

Definition $14([1])$. Let $K$ be a filter in $L$. A system $\mathscr{F}=\left\{A_{i} \in L^{X} \mid i \in I\right\}$ is called closed under $S_{K^{-}}$ intersections iff for each $A \in L^{U}$ it holds that

$$
\bigcap_{i \in I, S\left(A, A_{i}\right) \in K}\left(S\left(A, A_{i}\right) \rightarrow A_{i}\right) \in \mathscr{F}
$$

A system closed under $S_{K}$-intersections will be called an $L_{K}$-closure system. For $K=L$ the subscript will be omitted.

Remark 1. In general, being closed under arbitrary intersections -i.e. $\bigcap_{i \in J} A_{i} \in \mathscr{F}$ for all $J \subseteq I$ - is a weaker condition than being closed under $S_{K}$-intersections. See [1, Remark 3.3 (2)].

Our aim in this paper has been to highlight the definition of closure system on complete fuzzy lattices, not specifically over an $L$ powerset. Accordingly, the use of the external operations given in (2) are circumvented. To avoid confusion, we will rename Bělohlávek's $L$-closure systems as $S$-closure systems.

The following lemma is a technical result which is used in the proof of the next theorem. 
Lemma 15. Let $\left(L^{U}, S\right)$ be a L-powerset lattice. For all $A, B \in L^{U}$ we have that $A \subseteq S(A, B) \rightarrow B$ i.e. $S(A, S(A, B) \rightarrow B)=1$.

Proof. It is a consequence of $S(A, B) \otimes A(x) \leq B(x)$ for all $x \in U$.

Theorem 16. Let $\left(L^{U}, S\right)$ be a L-powerset lattice and $\mathscr{F} \subseteq L^{U}$. Then, $\mathscr{F}$ is a closure system if and only if $\mathscr{F}$ is an $S$-closure system. In addition, if $\mathscr{F}$ is a closure system then, for all $A \in L^{U}$,

$$
\min \left(A^{S} \cap \mathscr{F}\right)=\bigcap_{C \in \mathscr{F}}(S(A, C) \rightarrow C) .
$$

Proof. By Proposition 13, it is sufficient to prove that, in both cases, if $\mathscr{F}$ is a closure system or an $L$-closure system, the equality (3) holds.

On the one hand, assuming $\mathscr{F} \subseteq L^{U}$ is a closure system, let us prove (3). By Proposition 13 the element $M_{A}=\min \left(A^{S} \cap \mathscr{F}\right)$ exists, for all $A \in L^{U}$.

Since $M_{A}$ is a minimum it verifies $\left(A^{S} \cap \mathscr{F}\right)\left(M_{A}\right)=1$ and, for all $B \in L^{U},\left(A^{S} \cap \mathscr{F}\right)(B) \leq S\left(M_{A}, B\right)$. That is, $M_{A} \in \mathscr{F}, S\left(A, M_{A}\right)=1$ and, for all $C \in \mathscr{F}$, we have $S(A, C) \leq S\left(M_{A}, C\right)$.

Since $M_{A} \in \mathscr{F}$ and $S\left(A, M_{A}\right)=1$, we have that

$$
\bigcap_{C \in \mathscr{F}}(S(A, C) \rightarrow C) \subseteq S\left(A, M_{A}\right) \rightarrow M_{A}=M_{A} .
$$

Moreover, by Lemma 15 , and since $S(A, C) \leq S\left(M_{A}, C\right)$ for all $C \in \mathscr{F}$, we have that

$$
M_{A} \subseteq \bigcap_{C \in \mathscr{F}}\left(S\left(M_{A}, C\right) \rightarrow C\right) \subseteq \bigcap_{C \in \mathscr{F}}(S(A, C) \rightarrow C) .
$$

Thus, $\bigcap_{C \in \mathscr{F}}(S(A, C) \rightarrow C)=M_{A} \in \mathscr{F}$, for all $A \in L^{U}$ and $\mathscr{F}$ is an $S$-closure system.

On the other hand, assume $\mathscr{F}$ is an $S$-closure system, that is, $\bigcap_{C \in \mathscr{F}}(S(A, C) \rightarrow C) \in \mathscr{F}$ for all $A \in L^{U}$. Let $F_{A}=\bigcap_{C \in \mathscr{F}}(S(A, C) \rightarrow C)$ and prove $F_{A}=\min \left(A^{S} \cap\right.$ $\mathscr{F})$. First, $F_{A} \in \mathscr{F}$ and by Lemma 15 , we have that

$$
\begin{aligned}
S\left(A, F_{A}\right) & =S\left(A, \bigcap_{C \in \mathscr{F}}(S(A, C) \rightarrow C)\right)= \\
& =\bigwedge_{C \in \mathscr{F}} S(A, S(A, C) \rightarrow C)=1 .
\end{aligned}
$$

Finally, for all $C \in \mathscr{F}$, we have that $F_{A} \subseteq S(A, C) \rightarrow C$ and, therefore,

$$
S(A, C) \leq \bigwedge_{x \in U}\left(F_{A}(x) \rightarrow C(x)\right)=S\left(F_{A}, C\right) .
$$

Thus, for all $A \in L^{U}, F_{A}=\min \left(A^{S} \cap \mathscr{F}\right)$, hence $\mathscr{F}$ is a closure system.
Thus, in an $L$-powerset lattice $\left(L^{U}, S\right)$, a closure system is a crisp subset $\mathscr{F} \subseteq L^{U}$ which is closed under arbitrary infima of fuzzy subsets $\left(X \in L^{\mathscr{F}}\right.$ implies $\left.\prod X \in \mathscr{F}\right)$. This implies that $\mathscr{F}$ is closed under arbitrary crisp intersections $(X \subseteq \mathscr{F}$ implies $\bigcap X \in \mathscr{F})$. Nevertheless, as stated in Remark 1, the reciprocal implication does not hold. We illustrate it with the following example.

Example 2. Consider the universe set $U=\{a, b\}$, the lattice $L$ be the three-valued Łukasiewicz structure and $\left(L^{U}, S\right)$ the $L$-powerset lattice of $U$. Then, the set $\mathscr{F}=\{\{a / 1, b / 0.5\},\{a / 1, b / 1\}\}$ is a closure system. A fuzzy subset of $\mathscr{F}$ can be described as $X=$ $\{\{a / 1, b / 0.5\} / x,\{a / 1, b / 1\} / y\}$ where $x, y \in L$.

The infimum $\prod X$ is $\{a / 1, b / 0.5\}$ if $x=1$ and $\prod X=$ $\{a / 1, b / 1\}$ otherwise. Hence, $\mathscr{F}$ is a closure system.

As proved in Theorem 16, closure systems and $S$ closure systems are the same concept within $L$ powerset lattices. Let us show that $\mathscr{F}$ in this example is indeed an $S$-closure system .

In this particular case, $\bigcap_{C \in \mathscr{F}} S(A, C) \rightarrow C \in \mathscr{F}$ since for all $A \in L^{U}$ it is either $\{a / 1, b / 0.5\}$ or $\{a / 1, b / 0.5\}$.

Notice that these properties are stronger than being closed for arbitrary intersections. Consider the set $\mathscr{H}=\{\{a / 0, b / 0\},\{a / 1, b / 1\}\}$, this set is clearly closed under intersections but it does not satisfy the closure system nor the $S$-closure system definition.

Consider $A=\{a / 0.5, b / 0.5\}$, then

$$
\bigcap_{C \in \mathscr{H}} S(A, C) \rightarrow C=A \notin \mathscr{H} .
$$

Thus, $\mathscr{H}$ is not an $S$-closure system. Moreover, $\mathscr{H}$ is not a closure system either since for $B=$ $\{\{a / 0, b / 0\} / 0.5\} \subseteq \mathscr{H}$, we have $\left.\prod B=\{a / 0.5, b / 0.5\}\right) \notin \mathscr{H}$. Thus, $\mathscr{H}$ is not a closure system. The calculations of the infima and the intersections are left as an exercise to the reader.

\section{Fuzzy closure systems}

A natural generalisation of closure systems is to consider, instead of the set of closed elements, the degree to which each element is closed. For this aim, the equality between the element and its closure is replaced by the so-called $\otimes$-symmetric kernel relation, which is defined as $\left(a \approx_{\otimes} b\right)=\rho(a, b) \otimes \rho(b, a)$ for all $a, b \in A$. Thus, the degree in which an element $a \in A$ is closed is $\left(\mathrm{c}(a) \approx_{\otimes} a\right)$ and, since every closure operator is inflationary, it is equal to $\rho(\mathrm{c}(a), a)$. In [19], where the underlying structure is a Heyting algebra, the one-to-one relation between closure operators and 
fuzzy closure systems is easily obtained following the ideas used for closure systems. However, this is not the case when the underlying structure is a general residuated lattice case.

Theorem 17. Let $(A, \rho)$ be a complete fuzzy lattice and $\mathrm{c}$ be a closure operator on $\mathbb{A}$. For $\Phi_{\mathrm{c}} \in L^{A}$, defined as $\Phi_{\mathrm{c}}(a)=\rho(\mathrm{c}(a), a)$, we have that $\operatorname{Core}\left(\Phi_{\mathrm{c}}\right)=\mathscr{F}_{\mathrm{c}}$ is the closure system associated to $\mathrm{c}$ and, for all $x \in A$,

$$
\prod\left(x^{\rho} \otimes \Phi_{\mathrm{c}}\right)=\prod\left(x^{\rho} \cap \mathscr{F}_{\mathrm{c}}\right)
$$

In addition, for all $a \in A$, one has

$$
\Phi_{\mathrm{c}}(a)=\bigvee_{x \in \mathscr{F}_{\mathrm{c}}}\left(a \approx_{\otimes} x\right)
$$

Proof. Due to Theorem 7, to prove (4), it is enough to see that, for all $x, a \in A$,

$$
\begin{gathered}
\bigwedge_{y \in A}\left(\left(\rho(x, y) \otimes \Phi_{\mathrm{c}}(y)\right) \rightarrow \rho(a, y)\right) \\
=\bigwedge_{s \in \mathscr{F}_{c}}(\rho(x, s) \rightarrow \rho(a, s))
\end{gathered}
$$

On the one hand, the inequality $\leq$ is straightforward because $\rho(x, s) \otimes \Phi_{\mathrm{c}}(s)=\rho(x, s)$ for all $s \in \mathscr{F}_{\mathrm{c}}$. On the other hand, for all $y \in A$,

$$
\begin{aligned}
& \rho(x, y) \otimes \Phi_{\mathrm{c}}(y) \otimes(\rho(x, \mathrm{c}(y)) \rightarrow \rho(a, \mathrm{c}(y)))= \\
& =\rho(x, y) \otimes \rho(\mathrm{c}(y), y) \otimes(\rho(x, \mathrm{c}(y)) \rightarrow \rho(a, \mathrm{c}(y))) \\
& =\rho(x, y) \otimes \rho(y, \mathrm{c}(y)) \otimes \rho(\mathrm{c}(y), y) \\
& \otimes(\rho(x, \mathrm{c}(y)) \rightarrow \rho(a, \mathrm{c}(y))) \\
& \leq \rho(x, \mathrm{c}(y)) \otimes \rho(\mathrm{c}(y), y) \otimes(\rho(x, \mathrm{c}(y)) \rightarrow \rho(a, \mathrm{c}(y))) \\
& \leq \rho(a, \mathrm{c}(y)) \otimes \rho(\mathrm{c}(y), y) \leq \rho(a, y)
\end{aligned}
$$

Therefore, for all $a, x, y \in A$, we have that $\mathrm{c}(y) \in \mathscr{F}_{\mathrm{c}}$ and

$$
\rho(x, \mathrm{c}(y)) \rightarrow \rho(a, \mathrm{c}(y)) \leq\left(\rho(x, y) \otimes \Phi_{\mathrm{c}}(y)\right) \rightarrow \rho(a, y)
$$

Finally, we prove (5). For all $a \in A$, For all $a \in A$ and $x \in \mathscr{F}_{\mathrm{c}}$ (i.e. $\mathrm{c}(x)=x$ ), by isotony of $\mathrm{c}$ and transitivity, we have that $\left(a \approx_{\otimes} x\right)=\rho(a, x) \otimes \rho(x, a) \leq$ $\rho(\mathrm{c}(a), \mathrm{c}(x)) \otimes \rho(x, a) \leq \rho(\mathrm{c}(a), a)=\Phi_{\mathrm{c}}(a)$.

On the other hand, we have that $\mathrm{c}(a) \in \mathscr{F}_{\mathrm{c}}$ and $\Phi_{\mathrm{c}}(a)=\rho(\mathrm{c}(a), a)=\left(a \approx_{\otimes} \mathrm{c}(a)\right)$.

This leads to the definition of fuzzy closure systems.

Definition 18. Let $(A, \rho)$ be a complete fuzzy lattice. We say that a fuzzy set $\Phi \in L^{A}$ is a fuzzy closure system if $\operatorname{Core}(\Phi)$ is a closure system and, for all $a \in A$, the following condition holds

$$
\Phi(a)=\bigvee_{x \in \operatorname{Core}(\Phi)}\left(x \approx_{\otimes} a\right) .
$$

This additional property ensures that $\Phi$ is extensional.

Corollary 19. Let $\mathbb{A}=(A, \rho)$ be a complete fuzzy lattice. The following assertions hold:

i. If $\Phi$ is a fuzzy closure system on $\mathbb{A}$, the mapping $\mathrm{c}_{\Phi}: A \rightarrow A$ defined as $\mathrm{c}_{\Phi}(a)=\prod\left(a^{\rho} \otimes \Phi\right)$ is $a$ closure operator on $\mathbb{A}$.

ii. If $\mathrm{c}$ is a closure operator on $\mathbb{A}$, the fuzzy set $\Phi_{\mathrm{c}}$ defined as $\Phi_{\mathrm{c}}(a)=\rho(\mathrm{c}(a), a)$ is a fuzzy closure system on $\mathbb{A}$.

iii. If $\Phi$ is a fuzzy closure system on $\mathbb{A}$, then $\Phi=\Phi_{c_{\Phi}}$.

iv. If $\mathrm{c}: A \rightarrow A$ is a closure operator on $\mathbb{A}$, then $\mathrm{C}_{\Phi_{\mathrm{c}}}=\mathrm{c}$.

\section{Conclusions and future work}

In this paper, a version of the notion of closure system has been provided on general complete fuzzy lattices. This definition has been proved to behave properly in relation with closure operators, that is, there exists a bijection between both notions.

Moreover, the definition presented here has been compared with the original given by Bělohlávek, defined on $L$-powerset lattices, and have been proved to be equivalent. Hence, the former definition $\mathrm{c}$ an be used in general complete fuzzy lattices avoiding the explicit use of $L$-powerset tools, namely the external operations given in (2).

As prospect of future work, a next step might be the extension of the concept of closure operator and consider it as some kind of fuzzy relation instead of a crisp mapping, in line with the work in [7], and study the relationship between fuzzy closure systems and closure relations.

\section{Acknowledegment}

This work has been partially supported by the Spanish Ministry of Science, Innovation, and Universities (MCIU), the State Agency of Research (AEI), the Junta de Andalucía (JA), the Universidad de Málaga (UMA), and the European Social Fund (FEDER) through the FPU19/01467 internship (MCIU) and the research projects with reference TIN2017-89023-P, PGC2018-095869-B-I00 (MCIU/AEI/FEDER, UE) and UMA2018-FEDERJA001 (JA/UMA/FEDER, UE). 


\section{References}

[1] R. Bělohlávek, Fuzzy closure operators, Journal of Mathematical Analysis and Applications 262 (2001) 473-489.

[2] R. Bělohlávek, Fuzzy Relational Systems, Springer, 2002.

[3] R. Bělohlávek, Lattice type fuzzy order and closure operators in fuzzy ordered sets, in: Proceedings Joint 9th IFSA World Congress and 20th NAFIPS International Conference, Vol. 4, 2001, pp. 2281-2286 vol.4.

[4] R. Bělohlávek, B. De Baets, J. Outrata, V. Vychodil, Computing the lattice of all fixpoints of a fuzzy closure operator, IEEE Transactions on Fuzzy Systems 18 (3) (2010) 546-557.

[5] R. Bělohlávek, Concept lattices and order in fuzzy logic, Annals of Pure and Applied Logic 128 (1) (2004) 277-298.

[6] R. Bělohlávek, Pavelka-style fuzzy logic in retrospect and prospect, Fuzzy Sets and Systems 281 (2015) 61-72.

[7] I. Cabrera, P. Cordero, E. Muñoz-Velasco, M. Ojeda-Aciego, B. De Baets, Relational Galois connections between transitive fuzzy digraphs, Mathematical Methods in the Applied Sciences 43 (9) (2020) 5673-5680.

[8] I. P. Cabrera, P. Cordero, F. García-Pardo, M. Ojeda-Aciego, B. De Baets, On the construction of adjunctions between a fuzzy preposet and an unstructured set, Fuzzy Sets and Systems 320 (2017) 81-92.

[9] I. P. Cabrera, P. Cordero, F. García-Pardo, M. Ojeda-Aciego, B. De Baets, Galois connections between a fuzzy preordered structure and a general fuzzy structure, IEEE Trans. Fuzzy Systems 26 (3) (2018) 1274-1287.

[10] N. Caspard, B. Monjardet, The lattices of closure systems, closure operators, and implicational systems on a finite set: a survey, Discrete Applied Mathematics 127 (2) (2003) 241-269.

[11] P. Cordero, M. Enciso, A. Mora, V. Vychodil, Parameterized simplification logic I: reasoning with implications and classes of closure operators, International Journal of General Systems 49 (7) (2020) 724-746.

[12] B. De Baets, Analytical Solution Methods for Fuzzy Relational Equations, Springer US, Boston, MA, 2000, Ch. 6, pp. 291-340.
[13] J. Elorza, R. Fuentes-González, J. Bragard, P. Burillo, On the relation between fuzzy closing morphological operators, fuzzy consequence operators induced by fuzzy preorders and fuzzy closure and co-closure systems, Fuzzy Sets and Systems 218 (2013) 73-89.

[14] L. Guo, Q. Zhang, Q. Li, Fuzzy closure systems on L-ordered sets, Mathematical Logic Quarterly 57 (3) (2011) 281-291.

[15] P. Hájek, Metamathematics of Fuzzy Logic, Trends in Logic, Springer Netherlands, 2013.

[16] J. Konecny, M. Krupka, Complete relations on fuzzy complete lattices, Fuzzy Sets and Systems 320 (2017) 64-80.

[17] T. Kuhr, V. Vychodil, Fuzzy logic programming reduced to reasoning with attribute implications, Fuzzy Sets and Systems 262 (2015) 1-20.

[18] N. Madrid, J. Medina, M. Ojeda-Aciego, I. Perfilieva, Toward the use of fuzzy relations in the definition of mathematical morphology operators, Journal of Fuzzy Set Valued Analysis 2016 (1) (2016) $87-98$.

[19] M. Ojeda-Hernández, I. P. Cabrera, P. Cordero, E. Muñoz-Velasco, On (fuzzy) closure systems in complete fuzzy lattices, in: 2021 IEEE International Conference on Fuzzy Systems (FUZZIEEE), 2021, pp. 1-6.

[20] J. Poelmans, S. O. Kuznetsov, D. I. Ignatov, G. Dedene, Formal concept analysis in knowledge processing: A survey on models and techniques, Expert Systems with Applications 40 (16) (2013) 6601-6623.

[21] A. Tepavčević, G. Trajkovski, L-fuzzy lattices: an introduction, Fuzzy Sets and Systems 123 (2001) 209-216.

[22] B. Šešelja, A. Tepavčević, Fuzzifying closure systems and fuzzy lattices, Lecture Notes in Computer Sciences 4482 (2007) 111-118.

[23] B. Šešelja, A. Tepavčević, L-E-fuzzy lattices, International Journal of Fuzzy Systems 17 (2015) 366-374.

[24] W. Yao, L. X. Lu, Fuzzy Galois connections on fuzzy posets, Mathematical Logic Quarterly 55 (2009) 105-112. 